\title{
Co-expression of CDI47 (EMMPRIN), CD44v3-10, MDRI and monocarboxylate transporters is associated with prostate cancer drug resistance and progression
}

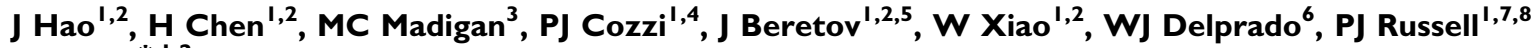 \\ and $\mathbf{Y} \mathbf{L i}^{*, 1,2}$
}

'Faculty of Medicine, UNSW, Kensington NSW 2052, Australia; ${ }^{2}$ Cancer Care Centre, St George Hospital, Gray St Kogarah, Kogarah NSW 2217, Australia; ${ }^{3}$ School of Optometry and Vision Science, UNSW, Kensington NSW 2052, Australia; ${ }^{4}$ Department of Surgery, St George Hospital, Kogarah, NSW 2217 Australia; ${ }^{5}$ Department of Anatomical Pathology, St George Hospital, Kogarah, NSW 2217, Australia; 'Douglass Hanly Moir Pathology, North Ryde, NSW 2113 Australia; ${ }^{7}$ Oncology Research Centre, Prince of Wales Clinical School, UNSW Randwick, NSW 203I, Australia; ${ }^{8}$ Australian Prostate Cancer Research Centre-Queensland, Institute of Health and Biomedical Innovation, Queensland University of Technology, 60 Musk Avenue, Kelvin Grove, QLD 4059, Australia

BACKGROUND: The aim of this study is to seek an association between markers of metastatic potential, drug resistance-related protein and monocarboxylate transporters in prostate cancer (CaP).

METHODS: We evaluated the expression of invasive markers (CDI47, CD44v3-I0), drug-resistance protein (MDRI) and monocarboxylate transporters (MCTI and MCT4) in CaP metastatic cell lines and CaP tissue microarrays ( $n=140$ ) by immunostaining. The co-expression of CDI47 and CD44v3-I0 with that of MDRI, MCTI and MCT4 in CaP cell lines was evaluated using confocal microscopy. The relationship between the expression of CDI47 and CD44v3-IO and the sensitivity (IC50) to docetaxel in CaP cell lines was assessed using MTT assay. The relationship between expression of CD44v3- I0, MDRI and MCT4 and various clinicopathological CaP progression parameters was examined.

RESULTS: CDI47 and CD44v3-10 were co-expressed with MDRI, MCTI and MCT4 in primary and metastatic CaP cells. Both CDI47 and CD44v3-10 expression levels were inversely related to docetaxel sensitivity (IC50) in metastatic CaP cell lines. Overexpression of CD44v3-10, MDRI and MCT4 was found in most primary CaP tissues, and was significantly associated with CaP progression.

CONCLUSIONS: Our results suggest that the overexpression of CDI47, CD44v3-I0, MDRI and MCT4 is associated with CaP progression. Expression of both CDI47 and CD44v3-I0 is correlated with drug resistance during CaP metastasis and could be a useful potential therapeutic target in advanced disease.

British Journal of Cancer (2010) I 03, 1008-1018. doi:I0.1038/sj.bjc.6605839 www.bjcancer.com

Published online 24 August 2010

(C) 2010 Cancer Research UK

Keywords: prostate cancer; CDI47; CD44v3- I0; monocarboxylate transporters; multidrug resistance; metastasis

Prostate cancer $(\mathrm{CaP})$ remains the most common cancer and the second leading cause of death from cancer in males in the United States (Jemal et al, 2009). Although early-stage CaP can be controlled using conventional therapies, multidrug resistance (MDR) and tumour metastasis remain the main causes of treatment failure and mortality in $\mathrm{CaP}$ patients. The majority of deaths in $\mathrm{CaP}$ result from progression to androgen-independent disease (Valdespino et al, 2007). For androgen-independent $\mathrm{CaP}$, chemotherapy is the standard treatment option for palliation of symptoms associated with the disease. However, the drug-resistant nature of $\mathrm{CaP}$ minimises therapeutic efficacy, and consequently, most patients die within 12 months. The relationship between

*Correspondence: Dr Y Li; E-mail: y.li@unsw.edu.au Revised I5 June 20 I0; accepted 12 July 20 I0; published online 24 August 2010 tumour metastasis and MDR is not fully defined in $\mathrm{CaP}$, although indirect evidence in advanced disease suggests a functional link between these processes

CD147 (EMMPRIN, extracellular matrix metalloproteinase inducer protein) is a multifunctional glycoprotein that can modify the tumour microenvironment by activating proteinases, inducing angiogenic factors in both tumour and stromal cells, and regulating growth and survival of anchorage-independent tumour cells (micrometastases) and MDR expression (Yan et al, 2005). CD147 is highly expressed on the surface of various tumours, including $\mathrm{CaP}$, and is associated with cancer progression (Riethdorf et al, 2006). Transcriptome analysis and comparative genomic hybridisation of individual tumour cells isolated from the bone marrow of patients with $\mathrm{CaP}$ have shown that $\mathrm{CD} 147$ is the most frequently expressed protein in primary tumours and micrometastases (Klein et al, 2002). Moreover, CD147 expression is considered a significant prognostic factor in human 
CaP (Zhong et al, 2008). Our recent results show that high-level CD147 expression is significantly correlated with CaP progression to high-grade disease, and is associated with the expression of MMPs in both tumour and stromal cells, including fibroblasts and endothelial cells (Madigan et al, 2008).

CD44 is a multifunctional protein involved in cell adhesion, migration and drug resistance. Alternative splicing of the CD44 gene produces many CD44 isoforms or variants (CD44v), some of which form the invariant extracellular domain of standard CD44 (CD44s). CD44s is expressed in the majority of normal basal prostate cells; however, CD44v expression is reported in $\mathrm{CaP}$ (Harrison et al, 2006). The role of $\mathrm{CD} 44$ in $\mathrm{CaP}$ development and progression is controversial, with studies showing both tumour-promoting and tumour-inhibiting effects (Gao et al, 1998; Omara-Opyene et al, 2004). Clearly the involvement of $\mathrm{CD} 44$ and its variants in $\mathrm{CaP}$ progression and metastasis is complex.

A major mechanism for drug resistance in cancer is through energy-dependent efflux pumps that reduce intracellular drug accumulation. One of these, which is well characterised, is MDR1/ P-glycoprotein (P-gp) or ABCB1, a 170-kDa membrane phosphoglycoprotein encoded by the $m d r 1$ gene (MDR1) (Germann, 1996). Previous studies indicated that CD147 expression is upregulated in MDR cancer cells, and also demonstrated that CD147 increases the activity of MMPs in MDR-expressing breast cancer cell lines (Yang et al, 2003). Treatment of MDR-expressing breast cancers with P-gp substrates can adversely affect therapeutic outcomes through modulation of CD147, MMP-2, MMP-9 and EGFR production ( $\mathrm{Li}$ et al, 2007). Hyaluronan (HA) production in mammary carcinoma cells is also increased by CD147, with MDR being induced in an HA-dependent manner (Marieb et al, 2004). Expression of CD44 and MDR1/P-gp seems to be co-regulated, as modulation of CD44 expression correspondingly affects MDR1/ P-gp expression in breast cancer (Miletti-González et al., 2005). Previous studies indicate that both CD44 and HA are involved in chemotherapeutic drug resistance in many cancer types (Misra et al, 2005; Ohashi et al, 2007), but regulation of MDR by CD147 and $\mathrm{CD} 44$ in $\mathrm{CaP}$ remains to be fully defined.

Tumour cell invasion and development of MDR are associated with hypoxia and low tumour $\mathrm{pH}$. Several studies show a direct relationship between increased cancer cell glucose uptake, glycolysis and tumour aggressiveness. Non-invasive spectroscopy imaging for hyperpolarised lactate also shows elevated lactate for high-grade $\mathrm{CaP}$ in a transgenic mouse model, compared with normal prostate (Albers et al, 2008). Tumour cell expression of MCT1 and MCT4 has been reported to be regulated by CD147 (Kirk et al, 2000). Specifically, interaction of CD147 with MCT1 or MCT4 within the endoplasmic reticulum is necessary for MCT trafficking to the plasma membrane; without CD147, MCTs are degraded and thus non-functional (Gallagher et al, 2007). However, the relationship of MCTs with CD147, CD44 and MDR1 in $\mathrm{CaP}$ is still unclear.

In this study, we investigated whether there is an association between markers of metastatic potential (CD147, CD44v3-10), MDR-related protein (MDR1) and monocarboxylate transporters (MCT1 and MCT4), and CaP progression and chemoresistance. We found colocalisation of CD147, CD44v3-10, MDR1, MCT1 and MCT4 in metastatic CaP cells lines; Expression of CD147 and CD44v3-10 in metastatic CaP cells was inversely related to docetaxel sensitivity $\left(\mathrm{IC}_{50}\right)$. In addition, we confirmed the colocalisation of CD147 and CD44v3-10 with MDR1, MCT1 and MCT4 in low- and high-grade primary $\mathrm{CaP}$ tissues, and demonstrated that overexpression of CD44v3-10, MDR1 and MCT4 is related to clincopathological markers of $\mathrm{CaP}$ progression. Our results suggest that $\mathrm{CD} 147$ and $\mathrm{CD} 44 \mathrm{v} 3-10$ are associated with $\mathrm{CaP}$ drug resistance and metastasis, and could be useful therapeutic targets to prevent the development of incurable, recurrent and drug-resistant $\mathrm{CaP}$.

\section{MATERIALS AND METHODS}

\section{Antibodies}

The following antibodies and conjugates were used: mouse antiEMMPRIN/CD147 monoclonal antibody (MAb) (8D6), rabbit polyclonal anti-human MDR1 antibody (sc-1517-R), rabbit polycolonal anti-MCT1 antibody (H-70), rabbit polycolonal anti-MCT4 (H-90) (Santa Cruz Biotechnology Inc, Santa Cruz, CA, USA); goat polyclonal anti-CD44v3-10 antibody (Alexis Biochemicals, San Diego, CA, USA); Alexa Fluor-488 goat anti-mouse IgG, Alexa Fluor-488 donkey anti-goat IgG, AlexaFluor-594 goat anti-rabbit IgG, Alexa Fluor-594 donkey anti-goat IgG (Molecular Probes, Eugene, OR, USA); biotinylated swine anti-goat, mouse, rabbit immunoglobulins (Igs), streptavidin/HRP, mouse anti-human IgG $_{1}$ negative control antibodies (Dako, Glostrup, Denmark); mouse anti-human MDR1 MAb (F4) mouse anti- $\beta$-tubulin MAb and goat or rabbit Ig (Sigma-Aldrich Pty Ltd, Castle Hills, NSW, Australia).

\section{Cell lines and cell culture}

Androgen-non-responsive (PC-3-RX-DT2R, PC-3, DU145) and androgen-responsive (LNCaP-LN3, DuCaP) $\mathrm{CaP}$ cell lines from different sources were studied (Supplementary Table 1s). Tissue culture reagents were supplemented with $10 \%(\mathrm{v} / \mathrm{v})$ heat-inactivated fetal bovine serum (Invitrogen Australia Pty Ltd, Melbourne, VIC, Australia), unless otherwise stated. PC-3-RX-DT2R, PC-3 and DU145 cells were cultured in RPMI-1640; LNCaP-LN3 cells in 1:1 RPMI-1640:F12-K; and DuCaP cells in DMEM. All were grown in a humidified incubator at $37^{\circ} \mathrm{C}$ with $5 \% \mathrm{CO}_{2}$. After $48 \mathrm{~h}$ culture, sub-confluent cells were rinsed twice with Dulbecco's phosphatebuffered saline (PBS) (pH 7.2), detached with $0.25 \%$ trypsin $/ 0.05 \%$ EDTA in PBS at $37^{\circ} \mathrm{C}$, collected by centrifugation and resuspended in buffer (see below).

The PC-3-RX-DT2R cell line was developed by Russell's group, by exposing xenografts of PC-3 to three doses of docetaxel at $12.5 \mathrm{mg} \mathrm{kg}^{-1}$ at 5-day intervals, intravenously, allowing the tumours to regress and then retreating the mice after their regrowth. Tumours that regrew after the second round of treatment were used to establish a line in culture. The cells were cultured in vitro with continuous exposure for 7 days to docetaxel at $1-1.25 \times 10^{-9} \mathrm{M}$, followed by a 14-day recovery period in the absence of added docetaxel through three rounds of treatment to establish them as a drug-resistant cell line, PC-3-RX-DT2R. DuCaP cells were provided by Dr K Pienta (University of Michigan Comprehensive Cancer Center, Ann Arbor, MI, USA). PC-3 and DU145 CaP cell lines were obtained from American Type Culture Collection (ATCC, Rockville, MD, USA). LNCaP-LN3 cells were kindly provided by Dr C Pettaway (M. D. Anderson Hospital, Austin, TX, USA).

\section{Immunofluorescence confocal microscopy analysis of CaP cell lines}

To determine the cellular localisation of CD147, CD44v3-10, MDR1, MCT1 and MCT4 in CaP cells, PC-3-RX-DT2R, PC-3, DU145, LNCaP-LN3 and DuCaP cells were grown on glass coverslips $\left(10^{5}\right.$ cells $)$ for $24 \mathrm{~h}$. After washing with Tris-buffered saline (TBS) ( $\mathrm{pH} \mathrm{7.5),} \mathrm{cells} \mathrm{were} \mathrm{fixed} \mathrm{on} \mathrm{coverslips} \mathrm{in} \mathrm{ice-cold}$ methanol for $10 \mathrm{~min}$ at room temperature (RT) and then incubated with $10 \%$ normal goat serum in TBS for 20 min to suppress nonspecific binding of IgG. After rinsing in TBS, the cells were incubated in mouse anti-CD147 (1:400 dilution), goat antiCD44v3-10 (1:400 dilution), rabbit anti-MDR1 (1:400 dilution), MCT1 (1:400 dilution) and rabbit anti-MCT4 (1:400 dilution) antibodies for $1 \mathrm{~h}$ at RT on a shaking table and rinsed with TBS, followed by a 45 -min incubation in Alexa Fluor-488 goat antimouse, donkey anti-goat or Alexa Fluor-594 goat anti-rabbit IgG (1:1000 dilution) at RT. The stained cells were mounted with 
glass slides using glycerol (Sigma-Aldrich Pty Ltd.). Slides were examined using an FV300/FV500 Olympus laser scanning confocal microscope (Olympus, Tokyo, Japan). Negative control slides were treated identically but with either isotype control MAbs or by omitting primary antibodies. We used a constant setting for laser power and detector gain for confocal microscopy. Multichannel excitation was minimised using fluorochromes with peak excitation of 488 and $594 \mathrm{~nm}$, respectively. Emission bleed-through was minimised using multitrack methods in which sequential image capture with a single detection channel was performed and images then combined. This corrects for the effects of emission crosstalk.

\section{Western blot analysis}

Protein expression levels were determined by western blot analysis. Briefly, cells were lysed in a buffer containing $50 \mathrm{mmoll}^{-1}$ Tris $-\mathrm{HCl}(\mathrm{pH} 8.0), 150 \mathrm{mmoll}^{-1}$ sodium chloride $(\mathrm{NaCl}), 0.1 \%$ SDS, $10 \mathrm{mmoll}^{-1}$ sodium fluoride $(\mathrm{NaF}), 1 \mathrm{mmoll}^{-1}$ sodium orthovanadate (Na3VO4), $0.5 \%$ sodium deoxycholate, $1 \%$ Triton $\mathrm{X}-100$ and $1 / 12(\mathrm{v} / \mathrm{v})$ protease inhibitor cocktail. The lysates were centrifuged at 13000 r.p.m. for $10 \mathrm{~min}$ at $4{ }^{\circ} \mathrm{C}$ and the supernatants were collected for determining protein concentration using a BCA protein assay reagent (Thermo Scientific, Waltham, MA, USA). Equal amounts of total protein were separated by NuPAGE 4-12\% Bis-Tris gel (Invitrogen Australia Pty Ltd) electrophoresis at $200 \mathrm{~V}$ for $50 \mathrm{~min}$ and then transferred to a PVDF membrane in NuPAGE transfer buffer at $30 \mathrm{~V}$ for $1.5 \mathrm{~h}$. Membranes were blocked with either $5 \%$ bovine serum albumin (SigmaAldrich Pty Ltd) or 5\% skim milk in PBS/0.05\% Tween 20 buffer. Blots were incubated overnight with specific antibodies at appropriate concentrations (CD147 1:300, CD44v3-10 1:100, mouse anti-MDR1 (F4) 1:500, MCT1 1:200 and MCT4 1:300 dilution) at $4{ }^{\circ} \mathrm{C}$. After washing for $3 \times 10 \mathrm{~min}$ in PBS $/ 0.05 \%$ Tween 20 buffer, blots were then incubated for another $1 \mathrm{~h}$ with an HRPconjugated IgG secondary antibody (Santa Cruz Biotechnology Inc, 1:5000 dilution). After washing for $3 \times 10 \mathrm{~min}$ in $\mathrm{PBS} / 0.05 \%$ Tween 20 buffer, immunoreactive bands were detected using ECL western blotting substrate (SuperSignal West pico Substrate, Thermo Scientific), followed by exposure to film and photographic development. To confirm equal loading of protein lysates, membranes were stripped (Restore Western Blot Stripping Buffer, Thermo Scientific) and re-probed using mouse anti- $\beta$-tubulin MAb (1:10000 dilution), then processed as above. Films were scanned and processed in Adobe Photoshop.

\section{MTT assay}

PC-3-RX-DT2R, PC-3, DU 145, LNCaP-LN3 and DuCaP cells were seeded in triplicate in 96-well plates at 5000 cells per well and incubated for $24 \mathrm{~h}$. A range of concentrations of docetaxel (SigmaAldrich, St Louis, MO, USA) diluted in $100 \%$ ethanol $(1000,100$, $10,1,0.1,0.01,0.001,0.0001 \mathrm{nM})$ was added to the cells. Control cells were treated with appropriate volumes of $100 \%$ ethanol. After $48 \mathrm{~h}, 20 \mu \mathrm{l}$ of MTT ( $5 \mathrm{mg} \mathrm{ml}^{-1}$ ) (Sigma-Aldrich Pty Ltd.) was added to each well, followed by incubation at $37^{\circ} \mathrm{C} / 5 \% \mathrm{CO}_{2}$ for $4 \mathrm{~h}$. Subsequently, $100 \mu \mathrm{L}$ of DMSO (Sigma-Aldrich Pty Ltd.) was added and the plate was shaken for $20 \mathrm{~min}$ at RT to dissolve the formazan crystals. The absorbance (OD) was read at a wavelength of $562 \mathrm{~nm}$ on a BIO-TEK microplate reader (Bio-Rad, Hercules, CA, USA). Each experiment was repeated at least three times. Results represent the OD ratio of treated and untreated cells. The growth inhibition curve was generated using the GraphPad Prism 4 Program (GraphPad, San Diego, CA, USA). Absolute IC $_{50}$ values were calculated using the intersection of the $50 \%$ normalised drug response and the growth inhibition curves for each cell line, to find the $x$ axis values for $\mathrm{IC}_{50}$ docetaxel concentration (nM) (also $\left.\log _{10}(\mathrm{~nm})\right)$.

\section{Patients and clinical data}

As described previously (Cozzi et al, 2006), $140 \mathrm{CaP}$ tissues were obtained with informed consent from patients with localised $\mathrm{CaP}$ undergoing radical resection of the prostate (RRP) or transurethral resection of the prostate at Urology Sydney, St George Private Hospital, from 2000 to 2007. Controls $(n=40)$ were from normal biopsy samples or from morphologically normal areas of $\mathrm{CaP}$ tissue. Ethical approval was obtained from the South East Area Health Human Research Ethics Committee, South Section. Specimens were grouped as follows: Group I: normal prostate glands (age $<40$ years, range $26-38$ years, $n=10$; age $>50$ years, range $=55-83$ years, $n=10$ ), normal areas of prostate glands from $\mathrm{CaP}$ patients (median age 67 years, range 62-84 years, $n=20$ ), benign prostate hyperplasia (BPH) (median age 66 years, range 58-72 years, $n=40)$, prostatic intraepithelial neoplasia (PIN) (median age 63 years, range 57-71 years, $n=20$ ); Group II: $120 \mathrm{CaP}$ specimens (96 RRP, 24 transurethral resection of the prostate), containing Gleason score $<7 \quad(n=30)$, Gleason score $=7 \quad(3+4) \quad(n=30)$, Gleason score $=7 \quad(4+3) \quad(n=30)$, Gleason score $>7(n=30)$, with median age 61 years (range $46-76$ years).

Formalin-fixed tissues were routinely processed, paraffinembedded and H\&E sections were reviewed. Tumour foci were identified, circled in ink and graded (Gleason system). Pathological stage (RRP) was determined using the TNM system. Clinical data in RRP patients $(n=96)$ indicated an average age at surgery of 63 years (range 49-72 years) and median follow-up time of 18 months (range 2-50 months). A detectable level of PSA $\left(>0.2 \mathrm{ng} \mathrm{ml}^{-1}\right)$ after surgery was defined as biochemical recurrence (Cozzi et al, 2006). Pertinent clinical information (pretreatment PSA level, Gleason score, clinical stage, surgical margin status, assessment by clinic visit, phone or e-mail contact to determine overall, cancer-specific and recurrence-free survival) was recorded. All patients were advised to undergo a serum PSA test twice a year.

\section{TMAs}

Tissue microarrays (TMAs) were constructed (Cozzi et al, 2006) with three tissue cores (diameter $1.0 \mathrm{~mm}$ )/donor block within the marked areas, being arrayed into a recipient paraffin block $(35 \mathrm{~mm} \times 20 \mathrm{~mm})$ of semiautomated Beecher Instruments (Silver Springs, MD, USA). Sections $(5 \mu \mathrm{m})$ were cut, collected on Superfrost Plus slides (Lomb Scientific, Sydney, NSW, Australia) and H\&E staining was performed.

\section{Immunohistochemistry}

To examine for expression of CD44v3-10, MDR1, MCT1 and MCT4 immunoreactivity, paraffin-embedded TMAs were deparaffinised in xylene, followed by a graded series of ethanol (100, 95, 75 and $50 \%$ ) and re-hydration in TBS. Slides were immersed in $0.01 \mathrm{M}$ citrate buffer ( $\mathrm{pH} 6.0)$ for $20 \mathrm{~min}$ at $100^{\circ} \mathrm{C}$ to enhance antigen retrieval, rinsed in TBS and then treated with $3 \%$ hydrogen peroxide, and again rinsed in TBS. After blocking in 10\% normal swine serum in TBS for $30 \mathrm{~min}$, sections were incubated overnight at $4{ }^{\circ} \mathrm{C}$ in goat anti-CD44v3-10 (1:200 dilution), rabbit anti-MDR1 (1:200 dilution), rabbit anti-MCT1 (1:200 dilution) and rabbit anti-MCT4 (1:200 dilution) Polyclonal antibodies (PAbs), washed in TBS, then incubated in biotinylated swine anti-goat or rabbit $\mathrm{Ig}(1: 150)$ for $45 \mathrm{~min}$ at $\mathrm{RT}$, rinsed in $\mathrm{TBS}$ and then incubated in streptavidin/HRP $(1: 200)$ for $30 \mathrm{~min}$ at RT. After rinsing in TBS, immunoreactivity was developed with $3,3^{\prime}$ diaminobenzidine substrate and counterstained with haematoxylin. Negative controls were treated identically but incubated in control PAbs (nonspecific goat or rabbit Ig), or the primary antibody was omitted. 


\section{Immunofluorescence staining of $\mathrm{CaP}$ tissues}

To examine the colocalisation of CD147, CD44v3-10, MDR, MCT1 and MCT4 in CaP tissues, whole sections (10 CaP specimens from each subgroup based on TMA immunohistochemistry results, $n=40$ ) were incubated overnight at $4{ }^{\circ} \mathrm{C}$ in primary mouse antiCD147 (1:200 dilution) MAb, or in goat anti-CD44v3-10 (1:200 dilution), rabbit anti-MDR1 (1:300 dilution), rabbit anti-MCT1 (1:200 dilution) and rabbit anti-MCT4 (1:200 dilution) PAbs. After washing with TBS, sections were incubated in goat antimouse Alexa 488 (CD147), goat anti-rabbit Alexa 594 (MDR1, MCT1 and MCT4) and donkey anti-goat Alexa 488 or 594 (for goat CD44v3-10) for $1 \mathrm{~h}$ at RT, and rinsed in TBS. Controls were treated identically, using nonspecific Igs (goat or rabbit Ig) as negative controls. Sections were examined using an FV 300/FV500 Olympus laser scanning confocal microscope (Olympus). We used a constant setting for laser power and detector gain for confocal microscopy, and multitracking and sequential image capture was used to correct signal emission crosstalk between neighbouring channels, and the images were combined.

\section{Assessment of immunostaining results}

Immunostaining results were assessed by staining intensity (Grade $0-3$ ) for cancer cell lines, TMA tissue and whole primary prostate and $\mathrm{CaP}$ tissues using light microscopy (Leica microscope, Nussloch, Germany) and confocal microscopy. The criteria for assessment were as follows: 0 , negative); 1 , weak); 2 , moderate); 3 , strong). For TMA staining, three cores were scored per case. The analysis of three cores per case has been shown to be comparable with the analysis of the whole section in a previous study (Rubin et al, 2002). In instances in which all three cores from one tumour were positive ( 3 of 3 ), the reading was counted as positive. In situations in which heterogeneous staining was seen among the three cores, an average score was determined. Evaluation of tissue staining was performed independently by three experienced observers (JLH, HMC and YL). All specimens were scored blind and an average of grades was taken. If discordant results were obtained, differences were resolved by joint review and consultation with a third observer (WD) experienced in immunopathology. For statistical analysis, CaP patients from RRP cases were divided into two groups: the low-expression group, comprising Grade 0 and 1 immunostaining, and the high-expression (overexpression) group, comprising Grade 2 and 3 immunostaining.

\section{Statistical analysis}

The associations between CD44v3-10, MDR1 and MCT4 expression levels (low-expression and high-expression groups) and clinicopathological data were tested using a $\chi^{2}$-test. Comparison of staining intensity for CD44v3-10, MDR1, MCT1 and MCT4 between $\mathrm{CaP}$ tissues and normal prostate tissues was performed using the $\chi^{2}$-test, where $P<0.05$ (two tailed) was considered significant. All statistical analyses were performed using GraphPad Prism 4.00 (GraphPad).

\section{RESULTS}

Expression and colocalisation of CD147, CD44v3-10, MDR1, MCT1 and MCT4 in metastatic and drug-resistance $\mathrm{CaP}$ cell lines

Immunofluorescence labelling of CaP cells with CD147, CD44v3-10, MDR1, MCT1 and MCT4 antibodies showed positive staining in PC-3-RX-DT2R, PC-3, DU145 and LNCaP-LN3 CaP cells, with variation between cell lines (Figure 1). Strong (Grade 3) expression of CD147, CD44v3-10, MDR1, MCT1 and MCT4 expression was found in PC-3-RX-DT2R and PC-3 cell lines. Medium expression of CD147, CD44v3-10, MDR1 and MCT1 and strong expression of MCT4 were found in DU145 cells. Low expression of CD147, CD44v3-10, MDR1 and MCT1 and medium expression of MCT4 were found in the LNCaP-LN3 cell line. DuCaP cells showed no staining for CD147 and CD44v3-10, and weak immunostaining for MDR1, MCT1 and MCT4. The immunostaining grades are summarised in Supplementary Table 2s. Membrane expression was found for CD44v3-10 PAb, whereas expression of both membrane and cytoplasm was seen for CD147, MDR1, MCT1 and MCT4 antibodies. Strong colocalisation of CD147/CD44v3-10, CD147/MDR1, CD147/MCT1, CD147/MCT4, CD44v3-10/MDR1, CD44v3-10/MCT1 and CD44v3-10/MCT4 was observed in PC-3RX-DT2R, PC-3 and DU 145 non-androgen-responsive metastatic $\mathrm{CaP}$ cell lines; weak colocalisation of these markers was observed in the LNCaP-LN3 cell line but no colocalisation was found in the $\mathrm{DuCaP}$ androgen-responsive $\mathrm{CaP}$ cell line (Figure 1 and Supplementary Figure 1s). The immunofluorescence results for the expression of CD147, CD44v3-10, MDR1, MCT1 and MCT4 in CaP cell lines were further confirmed by western blotting and high levels of these proteins were found in PC-3-RX-DT2R and PC-3 cell lines (Figure 2).

\section{Expression of CD147 and CD44v-3-10 is related with docetaxel response in metastatic $\mathrm{CaP}$ cell lines}

Metastatic CaP cell lines (PC-3-RX-DT2R, PC-3, DU145, LNCaPLN3 and DuCaP) with different levels of CD147 and CD44v3-10 expression responded differently to docetaxel treatment. The $\mathrm{IC}_{50}$ values for these $\mathrm{CaP}$ cell lines were highly related to the levels of CD147 and CD44v3-10 expression (see Figure 3 and Supplementary Table 2s). Thus, PC-3-RX-DT2R drug-resistant cells (CD147 and CD44v3-10, Grade3) were the least sensitive ( $\mathrm{IC}_{50}: 44.7 \mathrm{~nm}$ ), whereas DuCaP cells (CD147 and CD44v3-10, Grade 0) were very sensitive to docetaxel treatment $\left(\mathrm{IC}_{50}: 4 \mathrm{~nm}\right)$.

\section{Expression of CD44v3-10, MDR1, MCT1 and MCT4 in $\mathrm{CaP}$ tissues}

We previously reported CD147 expression in these TMAs (Madigan et al, 2008). In this study, we immunostained for CD44v3-10, MDR1, MCT1 and MCT4. In primary CaP tissues, 74 ( 89 of 120), 78 (94 of 120), 88 (106 of 120 ) and 92\% (110 of 120) were positive for CD44v3-10, MDR1, MCT1 and MCT4 (Grade $1-3)$, respectively. In CD44v3-10-positive primary $\mathrm{CaP}$ sections, weak staining (Grade 1) was found in 16\% (14 of 89) (Figure 4A), moderate staining (Grade 2) in 45\% (40 of 89) (Figure 4B) and strong staining (Grade 3) in 39\% (35 of 89) (Figure 4C), whereas no staining was observed in negative controls (Figure 4D).

In MDR1-positive primary $\mathrm{CaP}$ sections, weak staining (Grade 1) was found in 17\% (16 of 94) (Figure 4E), moderate staining (Grade 2) in 44\% (41 of 94) (Figure 4F) and strong staining (Grade 3) in 39\% (37 of 94) (Figure 4G), whereas no staining was found in negative controls (Figure $4 \mathrm{H}$ ).

The immunostaining patterns and percentage of positive cells for MCT1 and MCT4 in TMAs were similar, and MCT4 results are presented as representative of this study. In MCT4-positive primary CaP sections, weak staining (Grade 1) was found in $20 \%$ (22 of 110) (Figure 4I), moderate staining (Grade 2) in 38\% (42 of 110) (Figure 4J) and strong staining (Grade 3 ) in $42 \%$ (46 of 110) (Figure $4 \mathrm{~K}$ ), whereas no staining was found in negative controls (Figure 4L).

No CD44v-3-10, MDR1, MCT1 and MCT4 immunostaining was found in normal prostate tissues and PIN and in non-tumour regions from primary $\mathrm{CaP}$ tissues (data not shown). Scattered areas of weak ( $\leqslant$ Grade 1$)$ heterogeneous epithelial cell staining were observed in 3\% (1 of 40) for MDR1, and in $5 \%$ ( 2 of 40 ) for MCT4 in BPH specimens (Supplementary Table 3s). The staining intensity and percentage of positive staining for 
A PC-3-RX-DT2R

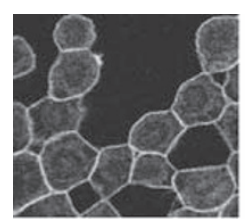

B

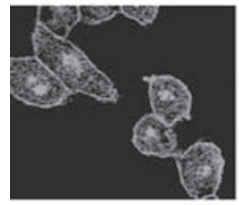

C

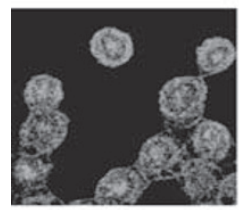

D

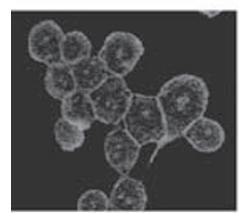

E

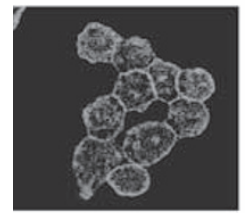

$\mathbf{F}$

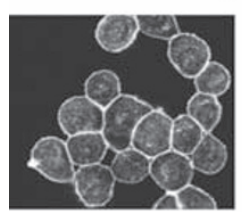

G

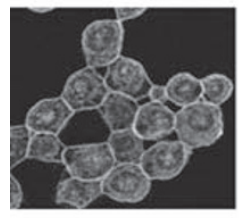

H

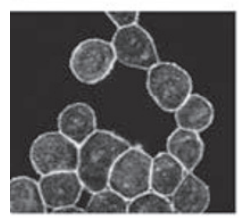

I

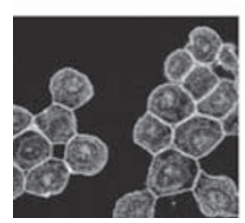

PC-3
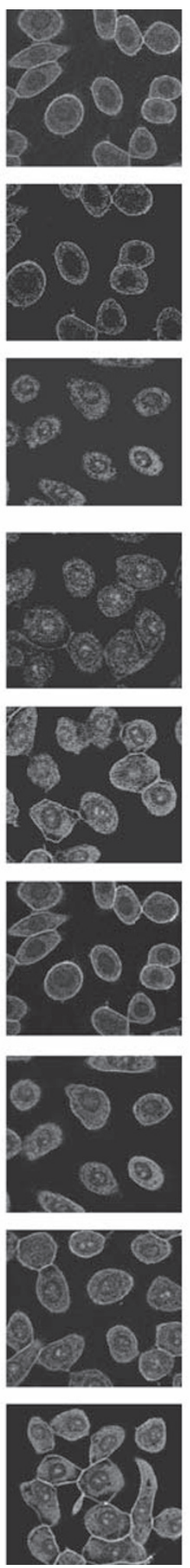

DU145
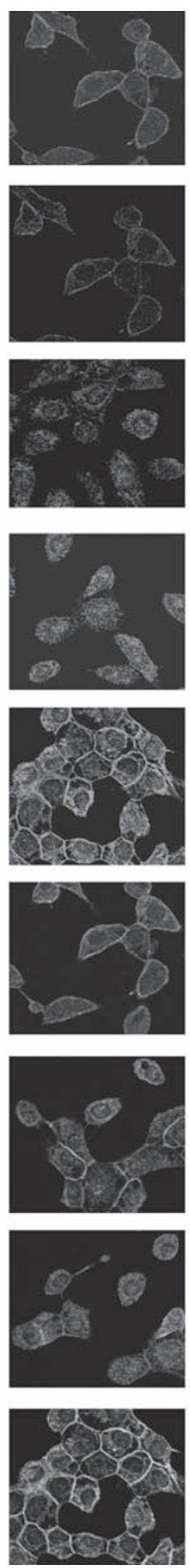

LNCaP-LN3
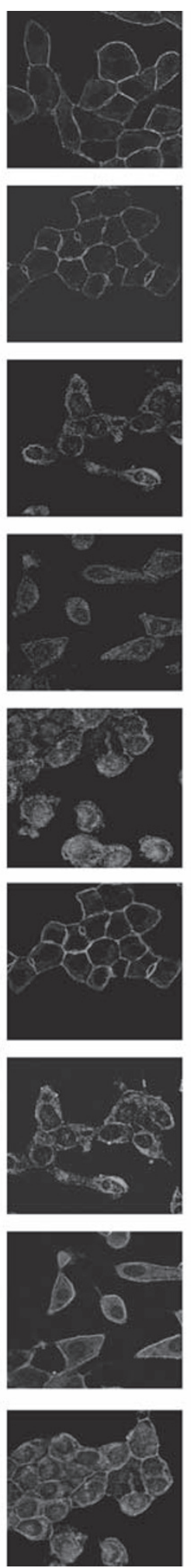

DuCaP

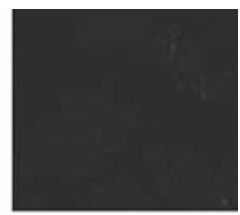

CD147

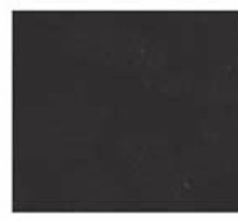

CD44v3-10

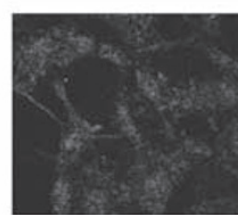

MDR1

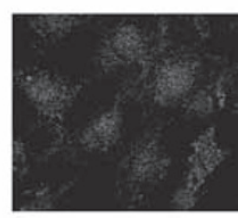

MCT1

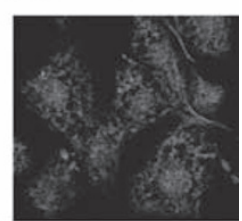

MCT4

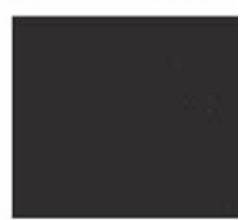

CD147/CD44v3-10

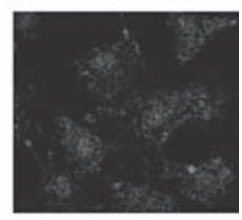

CD147/MDR1

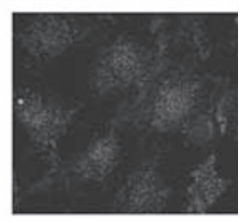

CD147/MCT1

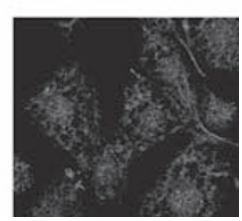

\section{CD147/MCT4}

Figure I Co-immunolabelling of CDI47, CD44v3-I0, MDRI, MCTI and MCT4 in metastatic prostate cancer (CaP) cell lines. Representative confocal images of CDI47 (green), CD44v3-I0, MDRI, MCTI and MCT4 (red) expression are shown. Merged images and red and green channels are shown separately. Membrane expression was found for CDI 47 and CD44v3-I0 antibodies, whereas expressions of both membrane and cytoplasm were seen for MDRI, MCTI and MCT4 antibodies. All immunostainings are more homogeneous. (A) CDI47; (B) CD44v3-I0; (C) MDRI; (D) MCTI; (E) MCT4; (F) colocalisation of CDI47 with CD44v3-I0; (G) colocalisation of CDI47 with MDRI; (H) colocalisation of CD I 47 with MCTI; (I) colocalisation of CD I 47 with MCTI. Magnification: A-I $\times 400$. The colour reproduction of the figure is available on the html full text version of the paper. 
CD44v3-10, MDR1, MCT1 and MCT4 in primary CaP tissues, PIN, BPH and normal prostates are summarised in Supplementary Table $3 \mathrm{~s}$ and Supplementary Table $4 \mathrm{~s}$. For CaP specimens, the immunostaining was mostly Grade 2 or 3, but was negative in PIN specimens.

Expression of CD44v3-10, MDR1, MCT1 and MCT4 was generally uniform in most tumours. The expression of CD44v3-10 was mostly cell membrane associated; however, distinct positive cytoplasmic staining was also seen. Immunostaining for MDR1, MCT1 and MCT4, as well as some membrane staining, was mainly cytoplasmic. In high-grade primary CaP (Gleason score $\geqslant 7$ ), the tumour stroma generally showed a strong positive reaction for CD44v3-10, MDR1, MCT1 and MCT4 (data not shown).

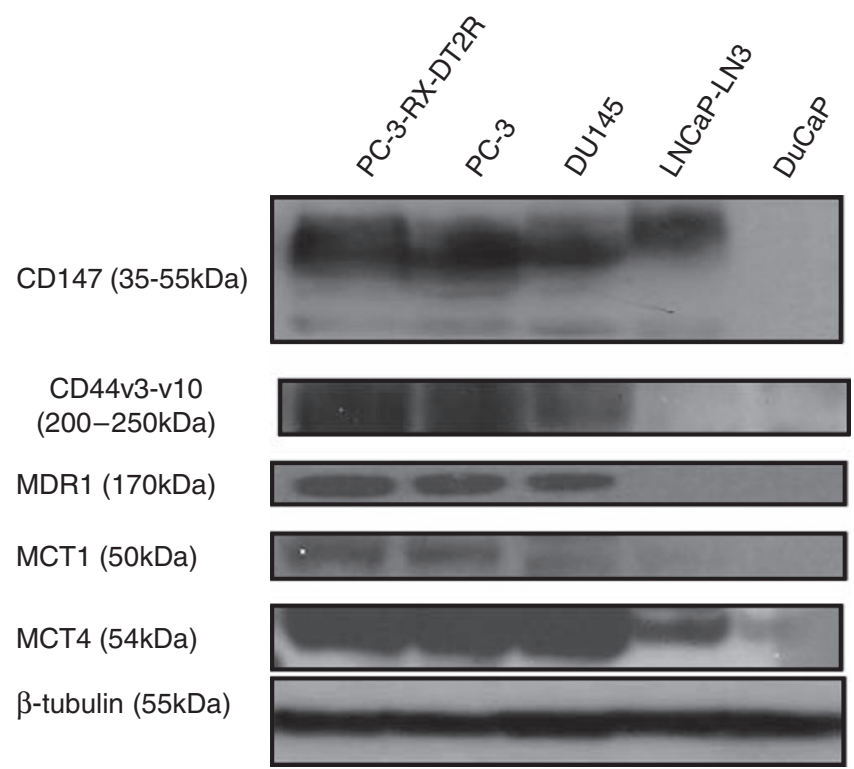

Figure 2 Expression of CDI47, CD44v3-I0, MDRI, MCTI and MCT4 in prostate cancer $(\mathrm{CaP})$ cell lines by western blotting. A representative western blot showing high levels of CDI47, CD44v3-I0, MDRI (F4), $\mathrm{MCTI}$ and MCT4 expression in PC-3-RX-DT2R and PC-3 cell lines, moderate expression in DU I 45 cells and low or no expression in LNCaP and $\mathrm{DuCaP}$ cells. Equal loading is demonstrated with $\beta$-tubulin antibody in the bottom panel (lane I: RX-DT2R, lane 2: PC-3, lane 3: DUI45, lane 4: LNCaP-LN3. lane 5: DuCaP).

\section{Correlation between CD44v3-7, MDR1 and MCT4 expression and clinicopathological parameters}

Of the 96 RRP patients, only 9\% (9 of 96) relapsed with biochemical progression (PSA $>0.4$ ), and no patients died of $\mathrm{CaP}$ during the follow-up period ( 5 years). The median time to relapse was 40 months (range 18-50 months). In all, $26 \%$ (25 of 96) of tumours had a Gleason score $<7$ whereas $74 \%$ (71 of 96) of tumours had a Gleason score $\geqslant 7$. In all, 27\% (26 of 96) of tumours were small (pT1), 38\% (36 of 96) were organ confined (stage pT2) and 36\% (34 of 96) had extracapsular extension (stage pT3). Table 1 summarises the correlations between CD44v3-10, MDR1 and MCT4 expression in primary CaP with a pretreatment PSA level, Gleason score, pathological stage, surgical margin status, nodal involvement (development of metastases) and biochemical recurrence. Overexpression (high-expression group) of CD44v3-10, MDR1 and MCT4 was significantly correlated with pretreatment PSA levels $(P<0.05)$, and increased with progression of $\mathrm{CaP}$ (Gleason score, $P<0.05$; pathological stage, $P<0.05$; nodal involvement, $P<0.05)$. Overexpression of CD44v3-10 and MDR1, but not of MCT4, was also significantly correlated with PSA-defined recurrence $(P<0.05)$. There was no correlation between overexpression of CD44v3-10, MDR1 or MCT4 and surgical margin $(P>0.05)$.

\section{Co-immunolabelling of primary CaP tissues with CD147, CD44, MDR1, MCT1 and MCT4 antibodies}

Colocalisation of CD147 and CD44v3-10, MDR1, MCT1and MCT4 was also assessed in primary CaP tissues $(n=40)$ by confocal microscopy. Most samples displayed co-immunolabelling with two different markers, although immunostaining for single antibody in different samples was variable. The immunostaining patterns are very similar to those seen by peroxidase immunohistochemistry as described above. Representative images from different tumours are shown in Figure 5. For co-immunolabelling of CD147 with CD44v3-10 (Figure 5A), CD147 with MDR1 (Figure 5B), CD147 with MCT1 (Figure 5C), CD147 with MCT4 (Figure 5D), CD147 is green, whereas CD44v3-10, MDR1, MCT1 and MCT4 expression is red. For co-immunolabelling of CD44v3-10 with MDR1 (Figure 5E), MCT1 (Figure 5F) and MCT4 (Figure 5G), CD44v3-10 is green, whereas MDR1, MCT1 and MCT4 expression is red.

\section{DISCUSSION}

In this study, we examined the expression of CD147, CD44v3-10, MDR1, MCT1 and MCT4 in metastatic CaP cell lines, in primary

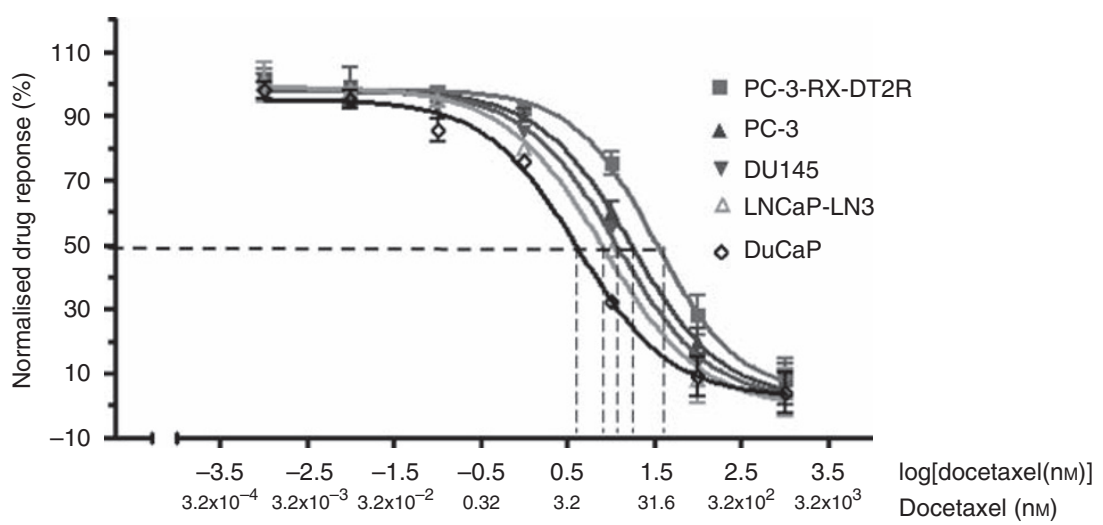

Figure 3 Dose response of metastatic prostate cancer (CaP) cell lines to docetaxel measured using 3-(4,5-dimethylthiazol-2-yl)-2,5-diphenyl tetrazolium bromide (MTT) assay. Drug-resistant and metastatic CaP cell lines treated with a range of concentrations of docetaxel $(0.001-1000 \mathrm{nM}, x$ axis $)$ showed varying responses. The $\mathrm{IC}_{50}$ value (50\% normalised cell response) is related to the expression of CDI47 and CD44v3-I0. For example, the drug-resistant cell line, PC3-RX-DT2R, displays Grade 3 CDI47 and CD44v3-10 expression, and an IC 50 of $44.7 \mathrm{~nm}$ ( $x$ axis); the drug-sensitive CaP cell line, DuCaP, displays Grade 0 CDI47 and CD44v3-I0 immunostaining and the lowest IC 50 of 4 nM ( $x$ axis) $(n=3$, mean \pm s.d.). 
1014
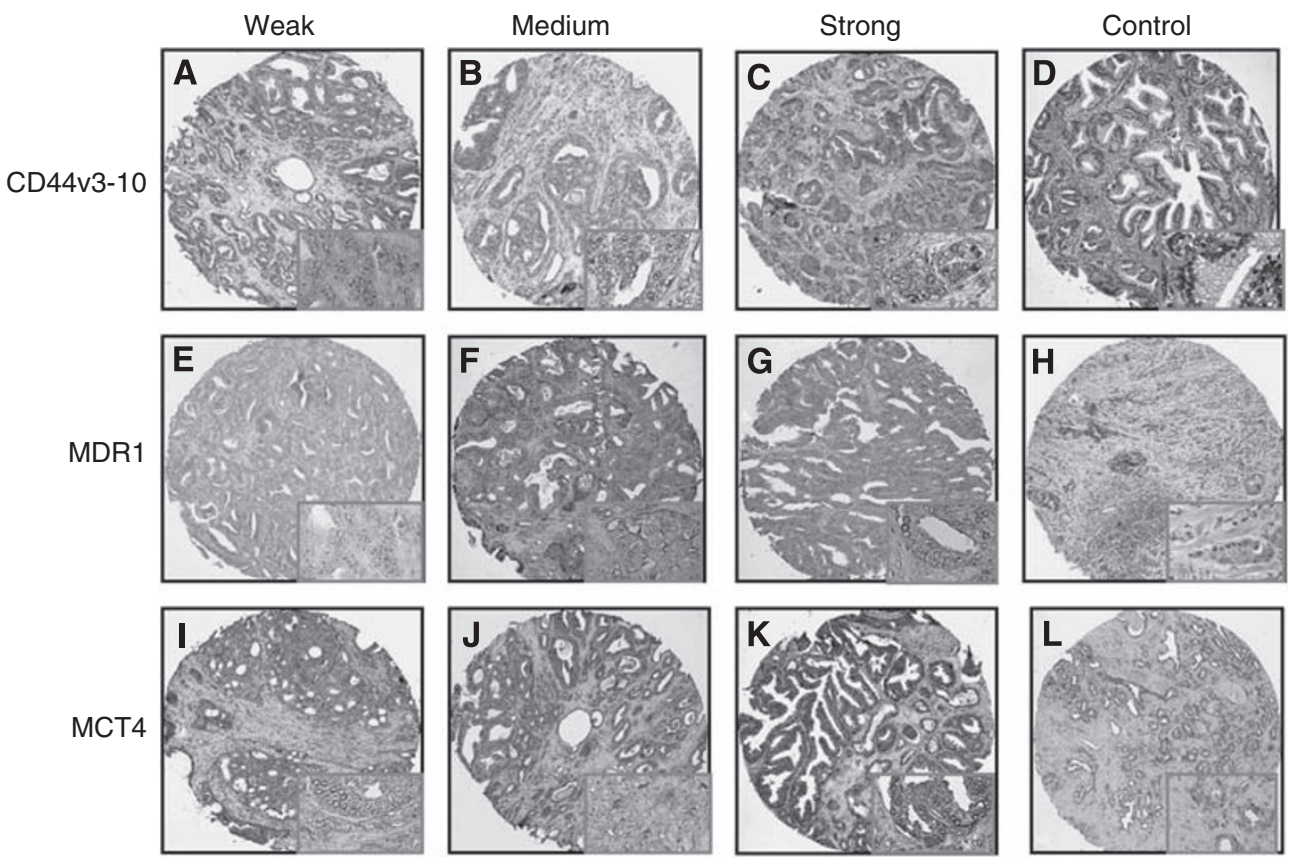

Figure 4 Expression of CD44v3- I0, MDRI and MCT4 in prostate cancer (CaP) tissue microarrays (TMAs). Representative images are shown of Grade I (weak) CD44v3-I0 (A), MDRI (E) and MCT4 (I); Grade 2 (medium) CD44v3-I0 (B), MDRI (F) and MCT4 (J); and Grade 3 (strong) CD44v3- I0 (C), $\operatorname{MDRI}(\mathbf{G})$ and MCT4 $(\mathbf{K})$ immunostaining. No immunoreactivity is seen in non-specific negative controls for CD44v3-I 0 (D)), MDRI (H) and MCT4 (L). Brown colour indicates positive immunostaining. Insets indicate the typical areas of staining at high amplification. Magnification: $\mathbf{A}-\mathbf{L} \times 10$; and insets $\times 40$. The colour reproduction of the figure is available on the html full text version of the paper.

Table I Clinicopathological characteristics associated with CD44v3-10, MDRI and MCT4 expression in primary CaPs (RRP patients, $n=96$ )

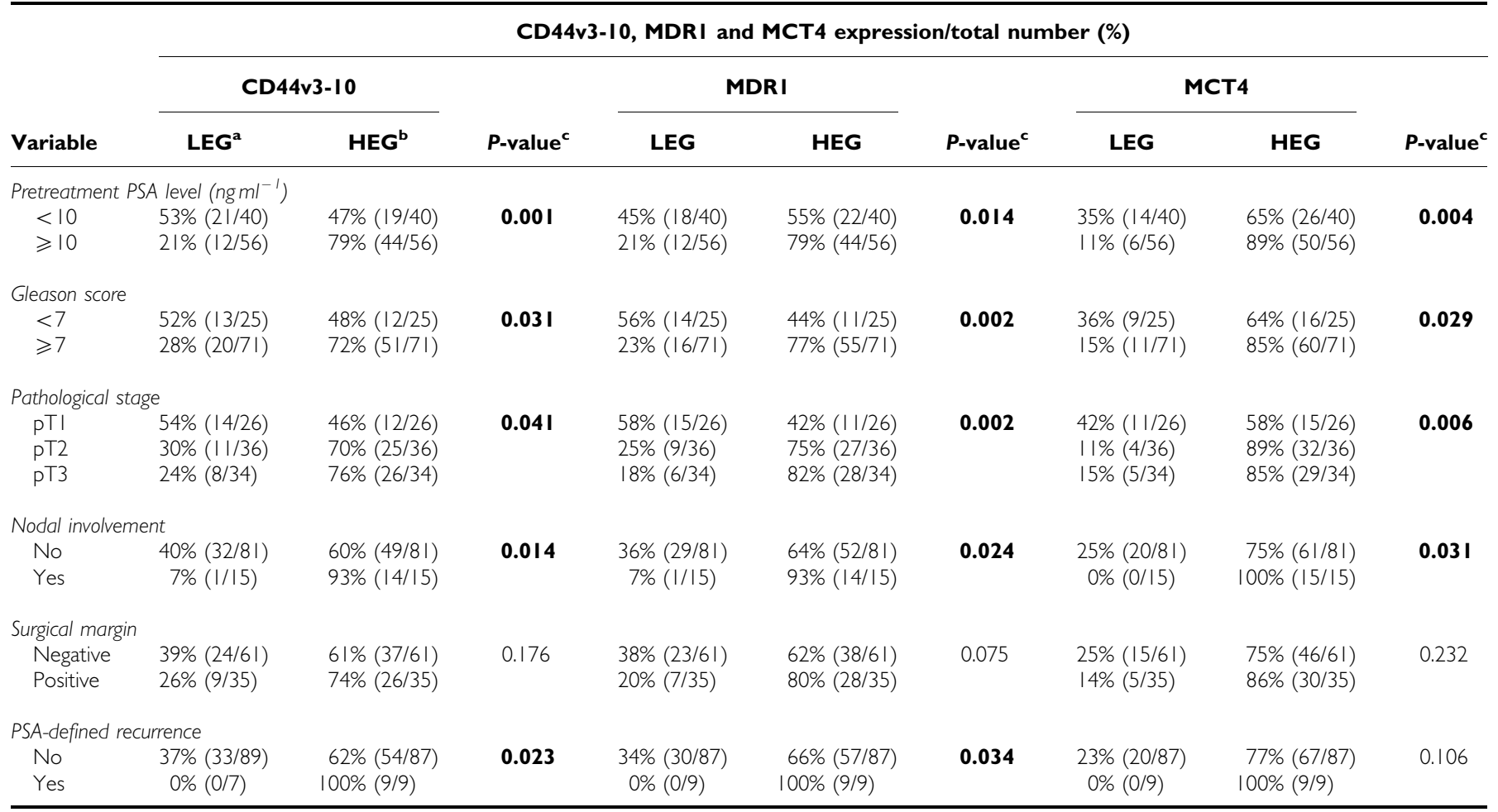

Abbreviations: $\mathrm{CaP}$, = prostate cancers; $\mathrm{HEG}=$ high expression group; LEG = low expression group; PSA = prostate-specific antigen; RRP = radical resection of the prostate. ${ }^{a} \mathrm{LEG}$ (Grade 0 or I immunostaining). ${ }^{b} \mathrm{HEG}$ ( $\geqslant$ Grade 2 immunostaining). ${ }^{c} \chi^{2}$-test; $P<0.05$ significant. The bold indicates the significant difference between groups in each parameter.

$\mathrm{CaP}$, PIN, BPH and normal prostate tissues using a tissue bank, and investigated further possible associations among these markers. High levels of CD147, CD44v3-10, MDR1, MCT1 and
MCT4 were observed in metastatic and drug-resistant CaP cell lines and in specimens of advanced CaP, but not in PIN, BPH and normal prostate tissues. Colocalisation of invasive and metastatic 
markers (CD147, CD44v3-10), MDR-related protein (MDR1) and monocarboxylate transporters (MCT1 and MCT4) was also found in most metastatic $\mathrm{CaP}$ cell lines, as well as in primary $\mathrm{CaP}$ tissues. To our knowledge, this is the first report investigating the
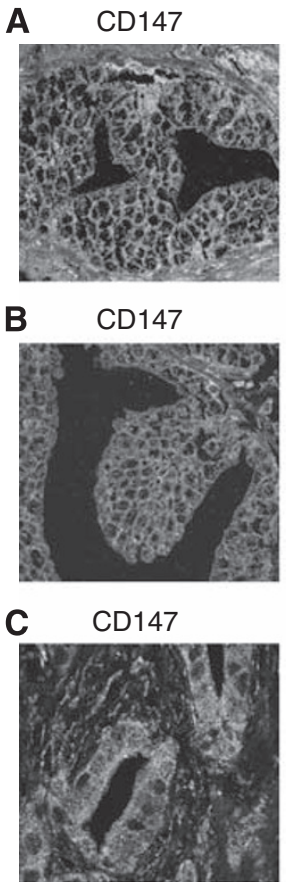

D

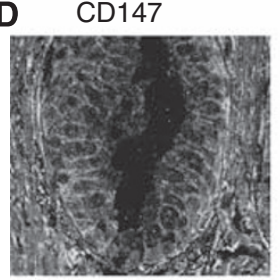

E CD44v3-10

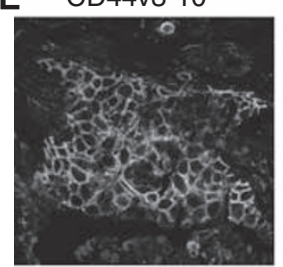

F $\quad$ CD44v3-10

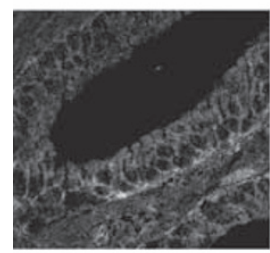

G CD44v3-10

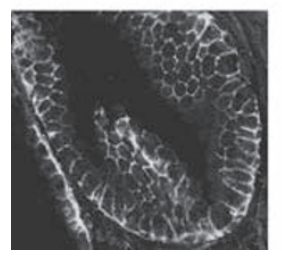

CD44v3-10

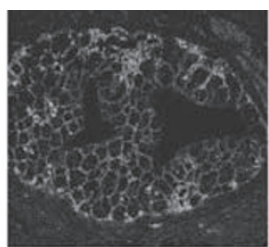

MDR1

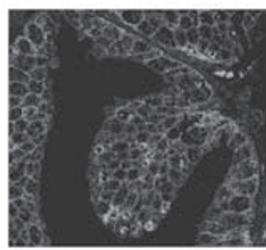

MCT1

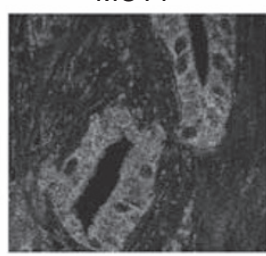

MCT4

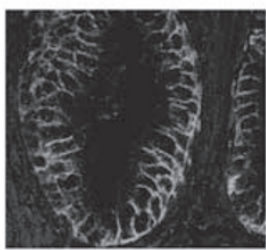

MDR1

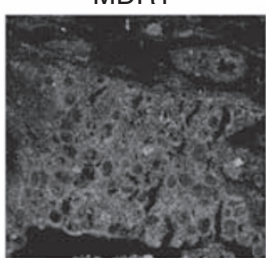

MCT1

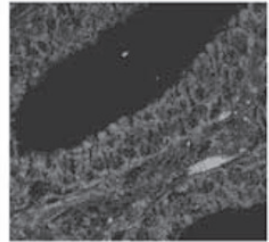

MCT4

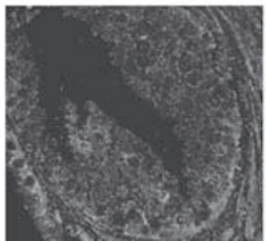

CD147/CD44v3-10

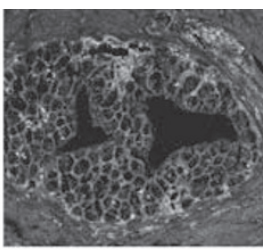

CD147/MDR1

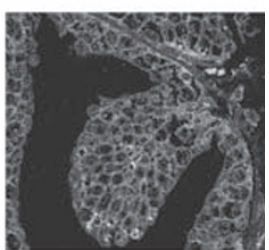

CD147/MCT1

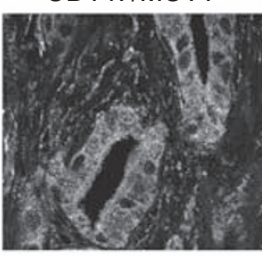

CD147/MCT4

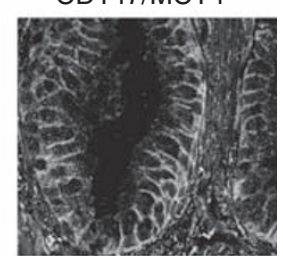

CD44v3-10/MDR1

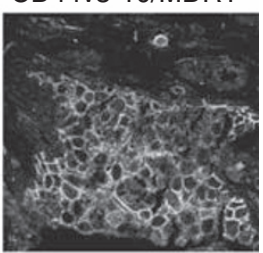

CD44v3-10/MCT1

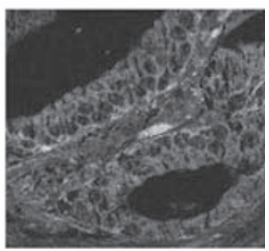

CD44v3-10/MCT4

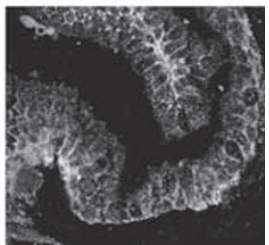

relationship between CD147, CD44v3-10, MDR1, MCT1 and MCT4 during $\mathrm{CaP}$ progression.

The colocalisation of CD147 separately with several different molecules, such as CD44v3-10, MDR1, MCT1 and MCT4, and the colocalisation of CD44v3-10 with MDR1, MCT1 and MCT4 in primary and metastatic CaP cells, suggests interactions between these proteins. Toole and Slomiany (2008) reported that CD147 and CD44 interact with various multidrug transporters of the $\mathrm{ABC}$ family and with MCTs associated with resistance to cancer therapies. Slomiany et al (2009) reported that CD44 colocalises with MCT1, MCT4 and CD147 at the plasma membrane, and HA, CD44 and CD147 contributed to the regulation of MCT localisation and function in the plasma membrane of breast cancer cells (Slomiany et al, 2009). Colocalisation of CD44 and MDR1 was shown to increase in melanoma cells engineered to express MDR, compared with parental cells (Colone et al, 2008). Su et al (2009) also found that CD147 colocalises with MCT1 and MCT4 in membranes of malignant A375 melanoma cells, leading to an increased glycolytic rate compared with that in normal human melanocytes. Silencing of CD147 in A375 cells abrogates expression of MCT1 and MCT4, and colocalisation with CD147, and dramatically decreases the cellular glycolytic rate, extracellular $\mathrm{pH}$ and the production of ATP (Su et al, 2009). Our present data show colocalisation of CD147 with MCT1 and MCT4 in primary and metastatic $\mathrm{CaP}$ cells, consistent with $\mathrm{CD} 147$ being an ancillary protein required for the expression of these MCTs (Deora et al, 2005; Gallagher et al, 2007). Our results support the hypothesis that expression of CD147 is closely related to that of CD44v3-10, and may be involved in regulating the expression of MDR1, MCT1 and MCT4 during $\mathrm{CaP}$ metastasis.

The expression of CD44 and its variants is associated with the progression of several cancers, although this remains controversial for CaP (De Marzo et al, 1998; Noordzij et al, 1999). One study reported a complete lack of membranous expression of all CD44 isoforms in $93-98 \%$ primary CaP tissues (Kallakury et al, 1996), whereas another reported moderate to high levels of CD44 expression in $60 \%$ of primary $\mathrm{CaP}$, with $\sim 14 \%$ of metastases expressing low levels of CD44 (Nagabhusha et al, 1996). Significant reduction in CD44 expression was also reported in primary $\mathrm{CaP}$ foci and metastases by De Marzo et al (1998). The relationship between CD44 expression and tumour grade is also uncertain, with a strong correlation between the Gleason grade of $\mathrm{CaP}$ and loss of CD44 expression in one study (De Marzo et al, 1998), but no correlation in another (Paradis et al, 1998). Similar to expression studies, the potential role of $\mathrm{CD} 44$ in $\mathrm{CaP}$ development and metastases is controversial. Earlier, overexpression experiments have suggested that CD44 may exert a tumour-suppressive function (Gao et al, 1998), although other studies have implicated $\mathrm{CD} 44$ in $\mathrm{CaP}$ cell proliferation, adhesion, migration and invasion

Figure 5 Co-immunolabelling of CDI47, CD44v3-I0, MDRI, MCTI and MCT4 in primary prostate cancer (CaP) tissues (different tumours with a range of Gleason scores). Representative confocal images of CDI47 and CD44v3-I0 (green), CD44v3-I0, MDRI, MCTI and MCT4 (red) immunolabelling are shown. Merged images and red and green channels are shown separately. (A) CDI47 and CD44v3-10 in high-grade $\mathrm{CaP}$ (Gleason score= 8); (B) CDI47 and MDRI in high-grade CaP (Gleason score = 8); $(\mathbf{C}) \mathrm{CDI} 47$ and MCTI in low-grade CaP (Gleason score = 6); (D) CD I 47 and MCT4 in high-grade CaP (Gleason score = 8); (E) CD44v3-10 and MDRI in high-grade CaP (Gleason score=9); (F) CD44v3-I0 and MCTI in high-grade CaP (Gleason score=8); $(\mathbf{G})$ CD44v3-10 and MCT4 in high-grade CaP (Gleason score=8). CDI47 immunolabelling is seen on epithelial cell membranes and stromal cells. CD44v3-I0, MDRI, MCTI and MCT4 immunostaining is predominantly epithelial. MCT4 localises mostly to basal epithelial cells and to lateral cell walls (magnification: $\mathbf{A}-\mathbf{G} \times 400$ ). The colour reproduction of the figure is available on the html full text version of the paper. 
in vitro, as well as in metastatic dissemination in vivo (Paradis et al, 1998; Omara-Opyene et al, 2004). The variation in CD44 expression seen in different studies may be attributable to the use of different methodologies in the assessment of CD44 expression or to the different stages of CaPs used in the analyses or to the use of different antibodies. Differences in the expressed CD44 isoform also explain some of these controversies. Non-invasive prostate epithelial cells have been shown to express a highmolecular-weight CD44 isoform, CD44v3-10, which may counteract the function of the standard isoform of CD44s by reducing adhesion to and invasion of the endothelium by $\mathrm{CaP}$ cells (Harrison et al, 2006).

In this study, we found CD44s expression in normal prostate tissues and in a very low percentage of cells in $\mathrm{CaP}$ tissues (of different stages, Hao J et al, unpublished data); CD44v3-10 was negative in all normal prostate and PIN tissues. However, high levels of expression of CD44v3-10 were correlated with tumour grade, clinical stage, residual tumour and relapse, but not with differences in tumour histological type. These observations support the idea that in the development of CaP, CD44 isoform expression changes progressively from CD44s to high-molecularweight variant forms such as CD44v3-10, and that CD44s basal cell expression is lost with overexpression of variant forms in $\mathrm{CaP}$ cells (Hao et al, 2010). The data suggest that CD44v3-10 is a marker of progression of prostate epithelial cells from a benign to a malignant phenotype, and thus may be an important indicator of the stage of $\mathrm{CaP}$, reflecting $\mathrm{CaP}$ progression and metastasis.

Aberrant MDR1 expression has been seen in many cancer types, including $\mathrm{CaP}$, and contributes significantly to treatment failure. MDR1 expression was found to be associated with drug resistance in androgen-dependent and androgen-independent human prostate xenografts (Chen et al, 1998), whereas downregulation of the MDR1 gene by hypermethylation has been associated with an increase in cellular proliferation possibly related to disease progression (Van Brussel et al, 2001; Enokida et al, 2004). In vitro studies have also reported a functional interaction between CD44 and MDR1, associated with increased cell migration, in vitro invasion and metastasis (Miletti-González et al., 2005). Our analysis of primary CaP tumour samples of different stages/ grades before drug therapy has shown high levels of MDR1 expression to be correlated with tumour grade, clinical stage, residual tumour and relapse, suggesting that MDR1 expression may be involved in CaP progression and metastasis. We also found that expression of CD147 and CD44v3-10 is colocalised in metastatic $\mathrm{CaP}$ cells and inversely related to docetaxel sensitivity in metastatic $\mathrm{CaP}$ cell lines, suggesting that CD147 and CD44v3-10 may be involved in $\mathrm{CaP}$ drug resistance. The functional roles of CD147 and CD44v3-10 in CaP metastasis and drug resistance are currently being investigated in our laboratory.

Increased glycolysis and adaptation to acidosis are key events in the transition from in situ to invasive cancer (Gatenby and Gillies, 2004). Given their essential function in exporting lactate, the end product of glycolysis, MCTs are considered key elements in regulating tumour intracellular $\mathrm{pH}$ and in the induction of extracellular acidosis (Pinheiro et al., 2009). The rapid transport of lactate through MCTs is of critical importance for tumour cells, by which an increased glycolytic rate gives a proliferative advantage over other cells. Upregulation of MCTs has been described in several tumour types, but only three studies have evaluated its clinicopathological significance (Pinheiro et al, 2008a,b, 2009). In this paper, we demonstrate for the first time that the high level of expression of MCT4 is correlated with $\mathrm{CaP}$ tumour grade, clinical stage and residual tumour, as well as with relapse, but not with differences in histological type, consistent with MCT1/MCT4 expression being involved in $\mathrm{CaP}$ progression.

We previously demonstrated CD147 expression in metastatic $\mathrm{CaP}$ cell lines, primary $\mathrm{CaP}$ tissues and lymph node metastases (Madigan et al, 2008). This has been ratified in this study, together with overexpression of CD44v3-10, MDR1, MCT1, MCT4 and colocalisation of CD147 and CD44v3-10, with MDR1, MCT1 and MCT4 in CaP and stromal cells (data not shown) in most primary tumours. The colocalisation of these markers in $\mathrm{CaP}$ tissues is consistent with that seen in $\mathrm{CaP}$ cell lines, suggesting that cancer clones that escape from primary tumours to the common metastatic sites in human $\mathrm{CaP}$ do not lose expression of these antigens. Differential expression of CD147, CD44v3-10, MDR1, MCT1 and MCT4 also suggests that the phenotypes of CaP metastasis may be controlled by genetics and/or by the tumour microenvironment during $\mathrm{CaP}$ progression. Functional interactions between CD44 and MDR1 are increasingly being recognised as important in tumour metastases. For example, in breast and ovarian cancer cell lines, immunoprecipitation and colocalisation studies, together with functional assays, showed that CD44 and MDR can directly influence the expression of each other, producing a malignant tumour cell phenotype characterised by MDR, increased migration and invasion (Miletti-González et al., 2005). The colocalisation of CD147 with CD44v3-10, MDR1, MCT1 and MCT4 in this study further suggests that CD147 and CD44v3-10 could concomitantly regulate MDR1, MCT1 and MCT4 expression during $\mathrm{CaP}$ progression, associated with drug resistance. However, the mechanisms involved in CD147 and CD44v3-10 regulation during $\mathrm{CaP}$ metastasis require further study. Given that CD147 and CD44v3-10 colocalise with MDR1-positive cells in $\mathrm{CaP}$ specimens, their targeting could potentially overcome drug resistance in the late stage of metastatic $\mathrm{CaP}$.

Previous studies have shown that CD147 knockdown using siRNA (Wang et al, 2006) or antibodies (Xu et al, 2007; Dean et al, 2009) inhibits tumour growth in vitro or in vivo, associated with changes in the regulation of MMP production (Xu et al, 2007, Dean et al, 2009) and radiation sensitivity of the tumours (Dean et al, 2009). Schneiderhan et al (2009) further confirmed that CD147 silencing inhibits lactate transport and reduces malignant potential of pancreatic cancer cells in in vitro and in vivo models. MCT1 inhibition has also been shown to have anti-tumour potential against in vivo models of lung carcinoma, colorectal carcinoma and a squamous carcinoma cell line after $\alpha$-cyano-4-hydroxycinnamate-mediated MCT1 inhibition (Sonveaux et al, 2008). These results suggest that targeting CD147 could be useful in controlling metastasis and cancer recurrence, with potential application to $\mathrm{CaP}$.

Targeting overexpressed CD44 in cancer cells may also control $\mathrm{CaP}$ progression. Antibody-mediated CD44 targeting has inhibited growth of breast cancer xenografts and prevented regrowth of basal-like $\mathrm{HBCx}$ cells after chemotherapy-induced remission (Marangoni et al, 2009). Gene therapy using siRNA CD44 also caused in vitro and in vivo regression of HT colon cancer cells (Subramaniam et al, 2007). Several reviews have also discussed the advantages of HA (major CD44 ligand) as a drug carrier and a targeting ligand for cancer, as well as other pathologies (Platt and Szoka, 2008; Yadav et al, 2008). It has also been used with prodrugs against cancer cell lines and xenografts (Auzenne et al, 2007) or in novel lipoplexes to target siRNA (Taetz et al, 2009), or for gene delivery (Surace et al, 2009). The potential for these approaches in $\mathrm{CaP}$, alone or in combination with CD147-targeted therapies (discussed above), is promising and remains to be investigated in future studies.

In summary, we have demonstrated co-expression of CD147 and CD44v3-10 with MDR1, MCT1 and MCT4 in most CaP metastatic cell lines and in primary $\mathrm{CaP}$ tissues. The overexpression of CD44v3-10, MDR1 and MCT4 was significantly associated with $\mathrm{CaP}$ progression. Colocalisation of CD147 and CD44v3-10 with MDR1 and MCTs in tumour and stromal cells suggests a role for these invasive markers in the regulation of drug resistance in the progression of $\mathrm{CaP}$, consistent with our in vitro docetaxel sensitivity findings. Our results further indicate that both CD147 and CD44v3-10 may be potential therapeutic targets for treating 
late-stage, incurable, recurrent metastatic $\mathrm{CaP}$ to overcome drug resistance.

\section{ACKNOWLEDGEMENTS}

The PC-3 docetaxel-resistant cell line was developed under a translational grant from the Cancer Institute of NSW. We thank Ms Elizabeth Kingsley, and Ms Mila Sajinovic, Oncology Research Centre, Prince of Wales Hospital, for their help in this development. We also thank Dr John Allen (Centenary Institute, University of Sydney, Australia) for kindly providing an MDR1-positive control cell line. This study was partially funded by the Career Development Fellowship from the Cancer Institute NSW, Australia (YL), and by the St George Hospital Trust Fund, Sydney, Australia.

Supplementary Information accompanies the paper on British Journal of Cancer website (http://www.nature.com/bjc)

\section{REFERENCES}

Albers MJ, Bok R, Chen AP, Cunningham CH, Zierhut ML, Zhang VY, Kohler SJ, Tropp J, Hurd RE, Yen YF, Nelson SJ, Vigneron DB, Kurhanewicz J (2008) Hyperpolarized 13C lactate, pyruvate, and alanine: noninvasive biomarkers for prostate cancer detection and grading. Cancer Res 68: 8607-8615

Auzenne E, Ghosh SC, Khodadadian M, Rivera B, Farquhar D, Price RE, Ravoori M, Kundra V, Freedman RS, Klostergaard J (2007) Hyaluronic acid-paclitaxel: antitumour efficacy against $\mathrm{CD} 44(+)$ human ovarian carcinoma xenografts. Neoplasia 9: 479-486

Chen CT, Gan Y, Au JL, Wientjes MG (1998) Androgen-dependent and -independent human prostate xenograft tumours as models for drug activity evaluation. Cancer Res 58: $2777-2783$

Colone M, Calcabrini A, Toccacieli L, Bozzuto G, Stringaro A, Gentile M, Cianfriglia M, Ciervo A, Caraglia M, Budillon A, Meo G, Arancia G, Molinari A (2008) The multidrug transporter P-glycoprotein: a mediator of melanoma invasion? J Invest Dermatol 128: 957 - 971

Cozzi PJ, Wang J, Delprado W, Madigan MC, Fairy S, Russell PJ, Li Y (2006) Evaluation of urokinase plasminogen activator and its receptor in different grades of human prostate cancer. Human Pathol 37: 1442 - 1451

Dean NR, Newman JR, Helman EE, Zhang W, Safavy S, Weeks DM, Cunningham M, Snyder LA, Tang Y, Yan L, McNally LR, Buchsbaum DJ, Rosenthal EL (2009) Anti-EMMPRIN monoclonal antibody as a novel agent for therapy of head and neck cancer. Clin Cancer Res 15: $4058-4065$

De Marzo AM, Bradshaw C, Sauvageot J, Epstein JI, Miller GJ (1998) CD44 and $\mathrm{CD} 44 \mathrm{v} 6$ downregulation in clinical prostatic carcinoma: relation to Gleason grade and cytoarchitecture. Prostate 34: $162-168$

Deora AA, Philp N, Hu J, Bok D, Rodriguez-Boulan E (2005) Mechanisms regulating tissue-specific polarity of monocarboxylate transporters and their chaperone CD147 in kidney and retinal epithelia. Proc Natl Acad Sci USA 102: 16245 - 16250

Enokida $\mathrm{H}$, Shiina $\mathrm{H}$, Igawa $\mathrm{M}$, Ogishima $\mathrm{T}$, Kawakami $\mathrm{T}$, Bassett $\mathrm{WW}$, Anast JW, Li LC, Urakami S, Terashima M, Verma M, Kawahara M, Nakagawa M, Kane CJ, Carroll PR, Dahiya R (2004) CpG hypermethylation of MDR1 gene contributes to the pathogenesis and progression of human prostate cancer. Cancer Res 64: 5956-5962

Gallagher SM, Castorino JJ, Wang D, Philp NJ (2007) Monocarboxylate transporter 4 regulates maturation and trafficking of CD147 to the plasma membrane in the metastatic breast cancer cell line MDA-MB-231. Cancer Res 67: 4182-4189

Gao AC, Lou W, Sleeman JP, Isaacs JT (1998) Metastasis suppression by the standard CD44 isoform does not require the binding of prostate cancer cells to hyaluronate. Cancer Res 58: 2350-2352

Gatenby RA, Gillies RJ (2004) Why do cancers have high aerobic glycolysis? Nat Rev Cancer 4: 891 - 899

Germann UA (1996) P-glycoprotein - a mediator of multidrug resistance in tumour cells. Eur J Cancer 32A: 927-944

Hao JL, Cozzi PJ, Khatri A, Power CA, Li Y (2010) CD147/EMMPRIN and $\mathrm{CD} 44$ are potential therapeutic targets for metastatic prostate cancer. Curr Cancer Drug Targ 10: 287-306

Harrison GM, Davies G, Martin TA, Mason MD, Jiang WG (2006) The influence of CD44v3-v10 on adhesion, invasion and MMP-14 expression in prostate cancer cells. Oncol Rep 15: 199-206

Jemal A, Siegel R, Ward E, Hao Y, Xu J, Thun MJ (2009) Cancer statistics, 2009. CA Cancer J Clin 59: 225-249

Kallakury BV, Yang F, Figge J, Smith KE, Kausik SJ, Tacy NJ, Fisher HA, Kaufman R, Figge H, Ross JS (1996) Decreased levels of CD44 protein and mRNA in prostate carcinoma. Correlation with tumour grade and ploidy. Cancer 78: $1461-1469$

Kirk P, Wilson MC, Heddle C, Brown MH, Barclay AN, Halestrap AP (2000) CD147 is tightly associated with lactate transporters MCT1 and MCT4 and facilitates their cell surface expression. EMBO J 19: 3896-3904

Klein CA, Seidl S, Petat-Dutter K, Petat-Dutter K, Offner S, Geigl JB, Schmidt-Kittler O, Wendler N, Passlick B, Huber RM, Schlimok G, Baeuerle PA, Riethmuller G (2002) Combined transcriptome and genome analysis of single micrometastatic cells. Nat Biotechnol 20: 387-392

Li QQ, Wang WJ, Xu JD, Cao XX, Chen Q, Yang JM, Xu ZD (2007) Involvement of $\mathrm{CD} 147$ in regulation of multidrug resistance to P-gp substrate drugs and in vitro invasion in breast cancer cells. Cancer Sci 98: $1064-1069$

Madigan MC, Kingsley EA, Cozzi PJ, Delprado WJ, Russell PJ, Li Y (2008) The role of extracellular matrix metalloproteinase inducer protein in prostate cancer progression. Cancer Immunol Immunother 57: $1367-1379$

Marangoni E, Lecomte N, Durand L, de Pinieux G., Decaudin D, Chomienne C, Smadja-Joffe F, Poupon MF (2009) CD44 targeting reduces tumour growth and prevents post-chemotherapy relapse of human breast cancers xenografts. Br J Cancer 100: 918-922

Marieb EA, Zoltan-Jones A, Li R, Misra S, Ghatak S, Cao J, Zucker S, Toole BP (2004) Emmprin promotes anchorage-independent growth in human mammary carcinoma cells by stimulating hyaluronan production. Cancer Res 64: 1229-1232

Miletti-González KE, Chen S, Muthukumaran N, Saglimbeni GN, Wu X, Yang J, Apolito K, Shih WJ, Hait WN, Rodríguez-Rodríguez L (2005) The CD44 receptor interacts with P-glycoprotein to promote cell migration and invasion in cancer. Cancer Res 65: 6660-6667

Misra S, Ghatak S, Toole BP (2005) Regulation of MDR1 expression and drug resistance by a positive feedback loop involving hyaluronan, phosphoinositide 3-kinase, and ErbB2. J Biol Chem 280: 20310-20315

Nagabhusha M, Pretlow TG, Guo YJ, Amini SB, Pretlow TP, Sy MS (1996) Altered expression of CD44 human prostate cancer during progression. Am J Clin Pathol 106: 647-651

Noordzij MA, van Steenbrugge GJ, Schroder FH, Van der Kwast TH (1999) Decreased expression of CD44 in metastatic prostate cancer. Int J Cancer 84: $478-483$

Ohashi R, Takahashi F, Cui R, Yoshioka M, Gu T, Sasaki S, Tominaga S, Nishio K, Tanabe KK, Takahashi K (2007) Interaction between CD44 and hyaluronate induces chemoresistance in non-small cell lung cancer cell. Cancer Lett 252: 225-234

Omara-Opyene AL, Qiu J, Shah GV, Iczkowski KA (2004) Prostate cancer invasion is influenced more by expression of a CD44 isoform including variant 9 than by Muc18. Lab Invest 84: 894-907

Paradis V, Eschwège $P$, Loric $S$, Dumas $F$, Ba N, Benoît G, Jardin A, Bedossa P (1998) De novo expression of CD44 in prostate carcinoma is correlated with systemic dissemination of prostate cancer. J Clin Pathol 51: $798-802$

Pinheiro C, Longatto-Filho A, Ferreira L, Pereira SM, Etlinger D, Moreira MA, Jube LF, Queiroz GS, Schmitt F, Baltazar F (2008a) Increasing expression of monocarboxylate transporters 1 and 4 along progression to invasive cervical carcinoma. Int J Gynec Pathol 27: 568-574

Pinheiro C, Longatto-Filho A, Scapulatempo C, Ferreira L, Martins S, Pellerin L, Rodrigues M, Alves VA, Schmitt F, Baltazar F (2008b) Increased expression of monocarboxylate transporters 1,2 and 4 in colorectal carcinomas. Virchows Arch 452: 139-146 
Pinheiro C, Longatto-Filho A, Simoes K, Jacob CE, Bresciani CJ, Zilberstein B, Cecconello I, Alves VA, Schmitt F, Baltazar F (2009) The prognostic value of CD147/EMMPRIN is associated with monocarboxylate transporter 1 co-expression in gastric cancer. Eur J Cancer 45: 2418-2424

Platt VM, Szoka Jr FC (2008) Anticancer therapeutics: targeting macromolecules and nanocarriers to hyaluronan or CD44, a hyaluronan receptor. Mol Pharm 5: 474-486

Riethdorf S, Reimers N, Assmann V, Kornfeld JW, Terracciano L, Sauter G, Pantel K (2006) High incidence of EMMPRIN expression in human tumours. Int J Cancer 119: 1800 - 1810

Rubin MA, Dunn R, Strawderman M, Pienta KJ (2002) Tissue microarray sampling strategy for prostate cancer biomarker analysis. Am J Surg Pathol 26: 312-319

Schneiderhan W, Scheler M, Holzmann KH, Marx M, Gschwend JE, Bucholz M, Gress TM, Seufferlein T, Adler G, Oswald F (2009) CD147 silencing inhibits lactate transport and reduces malignant potential of pancreatic cancer cells in in-vivo and in-vitro models. Gut 58: 1391 - 1398

Slomiany MG, Grass GD, Robertson AD, Yang XY, Maria BL, Beeson C, Toole BP (2009) Hyaluronan, CD44, and emmprin regulate lactate efflux and membrane localization of monocarboxylate transporters in human breast carcinoma cells. Cancer Res 69: 1293-1301

Sonveaux P, Vegran F, Schroeder T, Wergin MC, Verrax J, Rabbani ZN, De Saedeleer CJ, Kennedy KM, Diepart C, Jordan BF, Kelley MJ, Gallez B, Wahl ML, Feron O, Dewhirst MW (2008) Targeting lactate-fueled respiration selectively kills hypoxic tumour cells in mice. J Clin Invest 118: 3930 - 3942

Su J, Chen X, Kanekura T (2009) A CD147-targeting siRNA inhibits the proliferation, invasiveness, and VEGF production of human malignant melanoma cells by down-regulating glycolysis. Cancer Lett 273: 140-147

Subramaniam V, Vincent IR, Gilakjan M, Jothy S (2007) Suppression of human colon cancer tumours in nude mice by siRNA CD44 gene therapy. Exp Mol Pathol 83: 332-340

Surace C, Arpicco S, Dufaÿ-Wojcicki A, Marsaud V, Bouclier C, Clay D, Cattel L, Renoir JM, Fattal E (2009) Lipoplexes targeting the CD44 hyaluronic acid receptor for efficient transfection of breast cancer cell. Mol Pharm 6: $1062-1073$

Taetz S, Bochot A, Surace C, Arpicco S, Renoir JM, Schaefer UF, Marsaud V, Kerdine-Roemer S, Lehr CM, Fattal E (2009) Hyaluronic acid-modified DOTAP/DOPE liposomes for the targeted delivery of anti-telomerase siRNA to CD44-expressing lung cancer cells. Oligonucleotides 19: $103-116$

Toole BP, Slomiany MG (2008) Hyaluronan, CD44 and Emmprin: partners in cancer cell chemoresistance. Drug Resist Updat 11: 110-121

Valdespino V, Tsagozis P, Pisa P (2007) Current perspectives in the treatment of advanced prostate cancer. Med Oncol 24: 273-286

Van Brussel JP, Jan Van Steenbrugge G, Van Krimpen C, Bogdanowicz JF, Van Der Kwast TH, Schröder FH, Mickisch GH (2001) Expression of multidrug resistance related proteins and proliferative activity is increased in advanced clinical prostate cancer. J Urol 165: 130-135

Wang L, Wu G, Yu L, Yuan J, Fang F, Zhai Z, Wang F, Wang H (2006) Inhibition of CD147 expression reduces tumour cell invasion in human prostate cancer cell line via RNA interference. Cancer Biol Ther 5: 608-614

Xu J, Xu HY, Zhang Q, Song F, Jiang JL, Yang XM, Mi L, Wen N, Tian R, Wang L, Yao H, Feng Q, Zhang Y, Xing JL, Zhu P, Chen ZN (2007) HAb18G/CD147 functions in invasion and metastasis of hepatocellular carcinoma. Mol Cancer Res 5: 605-614

Yadav AK, Mishra P, Agrawal GP (2008) An insight on hyaluronic acid in drug targeting and drug delivery. J Drug Target 16: $91-107$

Yan L, Zucker S, Toole BP (2005) Roles of the multifunctional glycoprotein, emmprin (basigin; CD147), in tumour progression. Thromb Haemost 93: 199-204

Yang JM, Xu Z, Wu H, Zhu H, Wu X, Hait WN (2003) Overexpression of extracellular matrix metalloproteinase inducer in multidrug resistant cancer cells. Mol Cancer Res 1: 420-427

Zhong WD, Han ZD, He HC, Bi XC, Dai QS, Zhu G, Ye YK, Liang YX, Qin WJ, Zhang Z, Zeng GH, Chen ZN (2008) CD147, MMP-1, MMP-2 and MMP-9 protein expression as significant prognostic factors in human prostate cancer. Oncology 75: $230-236$ 